\title{
Las TICs en los procesos de enseñanza y aprendizaje
}

\section{TICs in teaching and learning processes}

Marco Antonio Gavilanes Sagñay. ${ }^{1}$, Willian Geovanny Yanza Chavez. ${ }^{2}$, Alex Fabián Inca Falconi. ${ }^{3}$, Germán Patricio Torres Guananga ${ }^{4} \&$ Roberto Fabian Sánchez Chávez ${ }^{5}$

\begin{abstract}
. DOI: https://doi.org/10.33262/cienciadigital.v3i2.6.575

The objective of this research was to analyze ICTs in the teaching-learning process of the university, so that a study is carried out on the evaluation made by teachers of a university in Ecuador on the advantages of using ICTs, in addition they identify the strategies used by them in the virtual campus. The methodology used was bibliographic, documentary and descriptive. Among the relevant results, it is observed that $100 \%$ of teachers give priority to the use of slide presentation programs, and only $66 \%$ use the Virtual platform, on the other hand, it is noticed that $54 \%$ of teachers participated in two training courses per year. Although it reveals a moderate interest of teachers for technical training, the accelerated development of educational technologies demands a greater dedication to the observed. The results allow to conclude that there is a predisposition to use techniques and learning tools, according to the intention to provoke creativity and innovation in the training exercise, which is related to the disciplinary areas of teacher performance. There is a greater propensity to explore technological tools in those disciplines that invest efforts in teacher training and the incorporation of these technologies.
\end{abstract}

KEYWORDS: ICTs, Teaching process, learning, virtual platform, education.

\footnotetext{
${ }^{1}$ Escuela Superior Politécnica de Chimborazo, Facultad de Administración de Empresas, Riobamba, Ecuador, magavilanes@yahoo.es

${ }^{2}$ Escuela Superior Politécnica de Chimborazo, Facultad de Administración de Empresas, Riobamba, Ecuador, willianyanza@hotmail.com

${ }^{3}$ Escuela Superior Politécnica de Chimborazo, Facultad de Administración de Empresas, Riobamba, Ecuador, alex.inca@espoch.edu.ec

${ }^{4}$ Escuela Superior Politécnica de Chimborazo, Facultad de Administración de Empresas, Riobamba, Ecuador, g_torres@espoch.edu.ec

${ }^{5}$ Escuela Superior Politécnica de Chimborazo, Facultad de Administración de Empresas, Riobamba, Ecuador, roberto.sanchez@espoch.edu.ec
} 


\section{RESUMEN.}

Esta investigación tuvo como objetivo analizar las TICs en el proceso de enseñanzaaprendizaje de la universidad, es así que se desarrolla un estudio sobre la valoración que hacen los docentes de una universidad de Ecuador sobre las ventajas del uso de las TICs, además se identifican las estrategias utilizadas por ellos en el campus virtual. La metodología utiliza fue bibliográfica, documental y descriptiva. Entre los resultados relevantes, se observa que el $100 \%$ de los docentes dan prioridad al uso de programas de presentación de diapositivas, y tan solo un $66 \%$ utiliza la plataforma Virtual, por otro lado, se advierte que el 54\% de los docentes participó en dos cursos de capacitación al año. Si bien revela un interés moderado de los docentes por la capacitación técnica, el desarrollo acelerado de las tecnologías educativas demanda una mayor dedicación a la observada. Los resultados permiten concluir que existe una predisposición de uso de técnicas y herramientas de aprendizaje, acorde a la intención de provocar la creatividad y la innovación en el ejercicio formativo, que se relaciona con las áreas disciplinares del desempeño docente. Se observa una mayor propensión a explorar las herramientas tecnológicas en aquellas disciplinas que invierten esfuerzos en la capacitación docente y en la incorporación de estas tecnologías.

PALABRAS CLAVES: TICs, Proceso de enseñanza, aprendizaje, plataforma virtual, educación.

\section{INTRODUCCIÓN.}

El Proceso de Enseñanza Aprendizaje (PEA) actualmente tiene como propósito contribuir a la formación del estudiante, a través del cumplimiento de objetivos instructivos y educativos. En la universidad, tradicionalmente, el profesor ha jugado un rol activo transmitiendo sus conocimientos. Pero el alumno ha ido adquirido un papel más dinámico en su formación y el profesor ha ido desarrollando sus funciones de forma menos activa. (Puig \& Ramos, 2009)

En la actualidad los procesos educativos en todo el mundo se enfrentan al desafío de utilizar las tecnologías de la información y la comunicación para proveer a sus alumnos con las herramientas y conocimientos necesarios que se requieren en el siglo XXI.

La UNESCO en el 2004 señala que en la educación, los objetivos estratégicos apuntan a mejorar la calidad de la educación por medio de la diversificación de contenidos y métodos, a través de la experimentación, la innovación, la difusión y el uso compartido de información y de buenas prácticas, la formación de comunidades de aprendizaje. 
Muchos de los aprendizajes de los estudiantes se logran gracias a los procesos de intercambio e interacción social, donde se permite construir y reconstruir conocimientos a través de estrategias colaborativas donde en escenarios virtuales surten un mayor sentido, ya que los procesos de comunicación y discusión requieren de un modelo que favorezca la interactividad colectiva. (Ruiz, 2012)

Con el advenimiento de las tecnologías de la información, el énfasis de la profesión docente está cambiando desde un enfoque centrado en el profesor que se basa en prácticas alrededor del pizarrón y el discurso, basado en clases magistrales, hacia una formación centrada principalmente en el alumno dentro de un entorno interactivo de aprendizaje.

El aprendizaje virtual se caracteriza por que no está confinado a la obligatoriedad del acto presencial de profesor y alumnos en una ubicación física en el aula, en un tiempo dado, y tiene el propósito substancial de que el alumno lo perciba con satisfacción y hasta como un entretenimiento, generando un efecto positivo en la tarea a realizar. (Kay \& Knaack, 2009)

En la educación superior los sistemas distribuidos asíncronos y de hipermedia presentan grandes oportunidades, tanto para los docentes como para los estudiantes, en términos de accesibilidad, de flexibilidad y, en algunos casos, de costos. Responder adecuadamente a cada uno de estos tres frentes mediante el uso de las TIC supone grandes desafíos para las instituciones, especialmente si al mismo tiempo se desea asegurar la calidad de estos procesos (Salinas, 2008).

De igual manera opinan (Palomo, Ruiz, \& Sánchez, 2010) quienes indican que las TIC ofrecen la posibilidad de interacción que pasa de una actitud pasiva por parte del estudiante a una actividad constante, a una búsqueda y replanteamiento continúo de contenidos y procedimientos. Aumentan la implicación del alumnado en sus tareas y desarrollan su iniciativa, ya que se ven obligados constantemente a tomar "pequeñas" decisiones, a filtrar información, a escoger y seleccionar.

Para que en la educación se puedan explotar los beneficios de las TICs en el proceso de aprendizaje, es esencial que tanto los futuros docentes como los docentes en actividad sepan utilizar estas herramientas. Para poder lograr un serio avance es necesario capacitar y actualizar al personal docente, además de equipar los espacios escolares con aparatos y auxiliares tecnológicos, como son pizarrones digitales interactivos, Proyectores, computadoras y conexión a la red.

La adecuación de profesores, alumnos, padres de familia y de la sociedad en general a este fenómeno, implica un esfuerzo y un rompimiento de estructuras para adaptarse a una nueva forma de vida; así, las universidades se podrían dedicar fundamentalmente a formar de manera integral a los individuos, mediante prácticas escolares acordes al desarrollo humano. 
En este orden de ideas, (Palomo, Ruiz, \& Sánchez, 2010) sostienen que las TIC se están convirtiendo poco a poco en un instrumento cada vez más indispensable en los centros educativos.

Asimismo estos autores señalan que estos recursos abren nuevas posibilidades para la docencia como por ejemplo el acceso inmediato a nuevas fuentes de información y recursos (en el caso de Internet se puede utilizar buscadores), de igual manera el acceso a nuevos canales de comunicación (correo electrónico, Chat, foros...) que permiten intercambiar trabajos, ideas, información diversa, procesadores de texto, editores de imágenes, de páginas Web, presentaciones multimedia, utilización de aplicaciones interactivas para el aprendizaje: recursos en páginas Web, visitas virtuales.

De igual manera tienen una serie de ventajas para el alumnado evidentes como: la posibilidad de interacción que ofrecen, por lo que se pasa de una actitud pasiva por parte del alumnado a una actividad constante, a una búsqueda y replanteamiento continuo de contenidos y procedimientos, también aumentan la implicación del alumnado en sus tareas y desarrollan su iniciativa, ya que se ven obligados constantemente a tomar "pequeñas" decisiones, a filtrar información, a escoger y seleccionar.

Es importante destacar que el uso de las TICs favorece el trabajo colaborativo con los iguales, el trabajo en grupo, no solamente por el hecho de tener que compartir ordenador con un compañero o compañera, sino por la necesidad de contar con los demás en la consecución exitosa de las tareas encomendadas por el profesorado. (Palomo, Ruiz, \& Sánchez, 2010).

\section{DESARROLLO}

Según (Adelle, 1997), "La relación del ser humano con la tecnología es compleja. Por un lado, la utilizamos para ampliar nuestros sentidos y capacidades. A diferencia de los animales, el ser humano transforma su entorno, adaptándolo a sus necesidades, las reales y las socialmente inducidas, pero termina transformándolo a él mismo y a la sociedad".

Existen cuatro etapas en el desarrollo de la comunicación:

El desarrollo del lenguaje oral. La primera fase que comienza con el desarrollo del lenguaje oral en los homínidos, esta fase se produce a lo largo de los siglos y dura aproximadamente 500.000 años-, una nueva dimensión a la interacción humana, directamente relacionada con el tiempo.

"La palabra hablada proporcionó un medio a los humanos de imponer una estructura al pensamiento y transmitirlo a otros". (Bosco, 1995). 
Este modo de transmisión de la información produce, (Marina, 1995), "una cultura oral, caracterizada por ser aditiva, agregativa, redundante, tradicionalista, centrada en la vida cotidiana, empática, situacional y participativa”. (Marina, 1995)

La difusión de la escritura. El uso de los signos gráficos para representar el habla, se produjo aproximadamente hace 3.500 años antes de nuestra era. La escritura permitió la independencia espacio-temporal entre el emisor y el receptor, y la acumulación y preservación de los conocimientos e informaciones para la posteridad.

Según (Comps, 1995), "la palabra escrita tenía algunos inconvenientes: era más lenta, más elitista; no todos las personas podían acceder a ella, y menos interactiva”.

La escritura estabilizó, despersonalizó y objetivizó el conocimiento, propiciando un pensamiento más reflexivo, sistemático, libre del contexto y estructurado. La utilización de la escritura como medio de transmisión de la información supone la necesidad de la alfabetización de las personas, creándose las primeras escuelas, cuyo objetivo era enseñar a los "escribas" la lectura y escritura.

La aparición de la imprenta. Con la aparición de la imprenta, con respecto a la fase anterior, tuvo consecuencias sociales, políticas, económicas, etc. de gran magnitud, que dieron lugar a la cultura moderna que todos conocemos. La imprenta permitió, una auténtica revolución en la difusión del conocimiento siendo accesible a todos y suprimiendo su carácter elitista. Sin lugar a duda, uno de los avances culturales y sociales más importantes. Los poderes políticos y sociales utilizan la información transmitida en los medios de comunicación impresos como un medio de propaganda y estrategia.

La influencia de la imprenta sobre el conocimiento y el pensamiento es revisada por (Bosco, 1995), para el que la estructura del libro se reproduce en la estructura de nuestro conocimiento. Este se caracteriza por ser lineal, estructurado en disciplinas cohesionadas, permanentes, acumulativas, ordenadas lógicamente, etc. Esto es, el modo en que conocemos determina el modo en que pensamos. La influencia de este pensamiento lineal se puede comprobar en los fundamentos pedagógicos y en los métodos de enseñanza utilizados.

"El concepto que usualmente tiende a manejarse de alfabetización informática es que el alumno domine algún lenguaje de programación, lo cual creo que es un error, ya que la alfabetización informática debe de perseguir objetivos más amplios, y me atrevería a decir que útiles: formación en una cultura general de las diversas actividades que pueden realizarse por medio del ordenador, formación en usos específicos de la informática, formación en su utilización como herramienta para la resolución de problemas, 
procesamiento y análisis de datos, hoja electrónica, formación en la cultura de la informática, limitaciones de los ordenadores, capacidad para manejar distintos programas..." (Cabero, 1996).

El uso de los medios electrónicos y la digitalización. Los avances en los medios electrónicos y la digitalización, y sobre todo la confluencia de los dos, han permitido crear entornos de comunicación totalmente nuevos. Estos entornos no están sujetos a un medio físico y en ellos la información se sitúa en un espacio no real a los que muchos autores han denominado "ciberespacio" o "espacio virtual", por lo que se dispone de posibilidades de transmisión de la información casi instantánea y a nivel global.

La gran influencia de estos medios se origina por el enorme impacto que presentan en todos los órdenes sociales (políticos, económicos, etc.), pero el resultado de la implantación de estas nuevas tecnologías dependerá en gran medida de los contextos sociales en los que se produzcan, y del uso que los ciudadanos hagan de las mismas. Internet como elemento más representativo de las TIC facilitan el tratamiento, creación, transferencia y comunicación de la información a nivel mundial. Al igual que ha ocurrido en las etapas anteriores, existe una fuerte pugna por el control de los nuevos medios, entre los poderes políticos, económicos y sociales.

Según (Adell, 1997) “... de todos estos desarrollos pueden obtenerse otros beneficios, del mismo modo que una red informática descentralizada, creada para soportar un ataque nuclear, se ha mostrado sumamente resistente a los intentos de censura y control ideológico de los gobiernos cuando ha pasado a las manos de los ciudadanos

\section{Funciones de las Tics en la educación}

La "sociedad de la información" en general y las nuevas tecnologías en particular, inciden de manera significativa en todos los niveles del mundo educativo. Las nuevas generaciones van asimilando de manera natural esta nueva cultura que se va conformando y que para nosotros conlleva muchas veces importantes esfuerzos de formación, de adaptación. En este sentido creemos que la universidad y los centros educativos en general, deben integrar también la nueva cultura: de alfabetización digital, fuente de información, instrumento de productividad para realizar trabajos, material didáctico, o como instrumento cognitivo, etc. En ese entendido la computadora se utiliza con finalidades diversas: lúdicas, informativas, comunicativas, instructivas.

Para lograr este cambio, se insta a los estudiantes a aprender a través de la estimulación de la vista, el oído, el tacto y mente; combinando texto, imágenes, sonido, animaciones y videos con la ayuda de la multimedia (Poole, 1999). 
Para (Mata, 1997), la educación debe formar a un alumno capaz de analizar, profundizar, buscar y encontrar el conocimiento. La labor del docente es ofrecer conocimientos abiertos al análisis, la reflexión, y al cambio, por tanto, la instrucción aplicable, debe tratar de adaptarse a cada individuo, pues presenta diversas habilidades, destrezas y capacidades cognoscitivas para aprender; la tarea del facilitador es saber reconocerlas y tratar de orientar sus estrategias y recursos hacia éstas.

\section{La nueva cultura implica el desarrollo de:}

- Medio de expresión (SOFTWARE): escribir, dibujar, presentaciones, webs... desde los que se encuentran en los paquetes de Windows, Paint, Word, en las XO o hasta programas que pueden ser encontrados en la web en portales como Wikinclusion.

- Canal de comunicación virtual (MENSAJERÍA, FOROS, WEBLOG, WIKIS, PLATAFORMAS e-CENTRO...), que facilita: trabajos en colaboración, intercambios, tutorías, compartir, poner en común, negociar significados, informar...

- Medio didáctico (SOFTWARE): informa, entrena, guía aprendizaje, evalúa, motiva. Hay muchos materiales interactivos autocorrectivos o de seguimiento de las tareas realizadas y los niveles alcanzados.

- Herramienta para la evaluación, diagnóstico y rehabilitación (SOFTWARE), adecuados a las capacidades diferentes de los estudiantes.

- Generador/Espacio de nuevos escenarios formativos. Multiplican los entornos y las oportunidades de aprendizaje contribuyendo a la formación continua en todo momento y lugar.

- Suelen resultar motivadoras (imágenes, vídeo, sonido, interactividad, etc). Y la motivación es uno de los motores del aprendizaje.

- Pueden facilitar la labor docente: más recursos para el tratamiento de la diversidad, facilidades para el seguimiento y evaluación (materiales autocorrectivos, plataformas), tutorías y contacto con las familias. Existen programas y periféricos (mouse adaptados, pulsadores, etc.) que facilitan acercamiento y el trabajo con la computadora.

\section{Uso de las TICs}

La introducción de las TICs en el ámbito educativo en los últimos años ha revolucionado los procesos educacionales; se han convertido en herramientas didácticas para el trabajo de educadores y educandos, haciendo más eficaz la gestión de los sistemas educativos. Internet brinda variados recursos que facilitan el acceso y procesamiento de la información susceptible de ser transformada en conocimiento. Estas tecnologías han democratizado la educación, dando oportunidades a todas las personas por igual, es un camino universal hacia 
el conocimiento (Organización de las Naciones Unidas para la Educación la Ciencia y la Cultura, 2017).

Existen diferencias en relación al uso que se ha realizado de las TICs en la modalidad de enseñanza presencial y la enseñanza a distancia, puesto que las características propias de cada una de las modalidades han llevado al uso de determinadas aplicaciones y servicios. No obstante, la influencia de la TICs ha dado lugar a sistemas de enseñanza semipresenciales (blendeg learning) que suponen cambios organizativos y estructurales de gran calado en relación a las enseñanzas presenciales y a distancia.

En la enseñanza presencial el uso de las TICs produce una ruptura de las limitaciones físicas del espacio, actuando como un espacio de comunicación e intercambio de información entre los miembros de la comunidad educativa. El uso pedagógico de las redes de comunicación puede propiciar que la relación entre educadores y educandos encuentre un ambiente que estimule la función del estudiante, como un agente activo de su propia instrucción, y la del maestro, como un guía más como una autoridad inapelable. En suma, Internet no solo provee herramientas, medios, recursos y contenidos sino, principalmente, entornos y ambientes que promueven interacciones y experiencias de interconexión e innovación educativa. El ordenador puede ser en el aula una ventana a la sociedad, en donde el alumno acceda a informaciones y contenidos relevantes para su formación.

De igual manera, el docente puede utilizar en su aula una fuente importante de recursos educativos de gran calidad y con un alto contenido multimedia, lo cual resulta extraordinariamente motivador para los alumnos. Por ejemplo, podemos acceder a páginas tan interesantes como la Bolsa de valores New York, la página web de la NASA, etc., muchas de las cuales disponen de apartados específicos destinados a los estudiantes. Sin embargo, se acentúa la necesidad de que el profesor realice una tarea como tutor, apoyando el proceso de aprendizaje, para que los alumnos realicen un aprendizaje constructivo, que les permita utilizar de una forma comprensiva y adecuada las informaciones a las que acceden, evitando el riesgo que plantea (Sculley, 1999), "Pienso que tenemos una sobrecarga de datos y, sin embargo, hay muy pocas personas que son capaces de utilizar la información de una forma satisfactoria" (pág. 167).

Según (ISTE, 1992) La Asociación Internacional para la Tecnología en la Educación, sostiene; "si lo que se pretende es formar adecuadamente a los estudiantes para que sean ciudadanos responsables en esta sociedad de la era de la información, es necesario que la tecnología informática sea una herramienta que tanto alumnos como profesores usen rutinariamente".

La introducción de los ordenadores en la educación produce una serie de efectos, que como indica (Collins, 1998), "favorecen el enfoque constructivista y en consecuencia la 
renovación del concepto de educación".

Este sistema ha tenido gran aceptación entre los estudiantes universitarios y en el mundo de la empresa, pues el estudiante no dispone de todo su tiempo por tener otras obligaciones, y puede adecuar el horario de formación a sus propias necesidades.

Así, el autor plantea los siguientes cambios:

- Cambio de la instrucción global a la instrucción individualizada. Observándose una reducción de las actividades dirigidas por el profesor del $70 \%$ al $10 \%$ cuando se utilizan los ordenadores en el aula.

- Cambio de la clase magistral y la exposición oral al entrenamiento y la instrucción. El uso de los ordenadores favorece que el profesor asuma el rol de instructor, encontrando un incremento del $20 \%$ al $50 \%$ en las actividades facilitadas por los profesores.

- Cambio de trabajar con los mejores alumnos a trabajar con los alumnos menos aventajados.

- Cambio de una evaluación basada en exámenes a una evaluación basada en productos, en el progreso y en el esfuerzo del alumno.

- Cambio de una estructura competitiva a una estructura cooperativa.

- Cambio de programas educativos homogéneos a la selección personal de contenidos.

- Cambio de la primacía del pensamiento verbal a la integración del pensamiento visual y verbal.

Según (Suasnabas, Díaz, Avila, \& Rodríguez, 2017) El uso de las TIC presenta ventajas en su comparación con los recursos utilizados en la enseñanza tradicional. La mayoría de estas ventajas están relacionadas directamente con las propias características de las TIC. Entre ellas cabe destacar: Sin lugar a duda, una de las potencialidades más importantes de Internet en el proceso de educación y formación de las personas, se produce en la enseñanza a distancia, convirtiéndose la red en la herramienta que, actualmente, facilita tremendamente el aprendizaje y la realización de cursos a distancia, hasta el punto de aproximar en gran medida la enseñanza a distancia a la enseñanza presencial (ej. Videoconferencias). La educación y/o formación por medios telemáticos ha tenido gran implantación para atender las necesidades de la educación continua y a distancia de las personas adultas. Puesto que, presenta las características y ventajas propias de la enseñanza a distancia, junto con las posibilidades de la intercomunicación a través de los servicios de Internet. Así:

Los alumnos que tienen dificultad para asistir a centros de enseñanza presencial, por vivir en zonas rurales alejadas de los mismos, o por sus características psicofísicas, pueden acceder a cursos de formación a través de la red desde sus propios domicilios. 
La comunicación entre tutor-alumno y entre los propios alumnos del curso mejora ampliamente en los cursos basados en la red, sobre los cursos de enseñanza a distancia tradicionales. Algunos autores hablan de un nuevo paradigma.

\section{Desafíos de las TICS para los cambios educativos}

Según (Prieto, y otros, 2011), "la humanidad se encuentra actualmente en el "punto de viraje" de una transformación tecnológica sin precedentes".

Las nuevas tecnologías vienen revolucionando además las percepciones del tiempo y del espacio; a su vez, Internet se revela intensamente social, desencadenando ondas de choque en el modo como las personas interactúan entre sí a una escala planetaria.

Por otro lado, estas tecnologías poseen una de las herramientas más importantes que constituye un hito tecnológico de gran impacto social, Internet; a través de sus recursos se tiene acceso a información de forma rápida, sencilla, eficiente y de calidad. Además, de ser una plataforma para compartir datos por medio de su interconectividad a nivel mundial, llegando a casi todos los rincones del mundo. Entre las características que favorecen su inclusión en los sistemas educacionales están: (Heinze, Olmedo, \& Andoney, 2017)

- Interconexión, facilita el acceso a información de cualquier red conectada a internet.

- Interactividad, permite la participación en múltiples redes sociales y de otra índole. Se pueden crear sitios Web de disímiles tipos: educativos, de servicios, etc.

- Instantaneidad, se refiere a la velocidad con la que se puede acceder a la información que está situada en el ciberespacio.

- Calidad de imagen y/o sonido, sus altos estándares permiten el consumo de productos audiovisuales y multimedios.

- Universalidad, está extendida por todo el mundo. Desde cualquier parte se puede acceder a información generada en lugares distantes, enviar correo y transferir archivos. Es posible conocer e interactuar con personas de todas partes del mundo.

- Económica, se ahorra tiempo al poder consultar una gran cantidad de información en menor lapso. Además de economizar los costos por concepto de envío de correspondencia y otros servicios.

"La utilización combinada de las tecnologías multimedia e Internet hace posible el aprendizaje en prácticamente cualquier escenario (la escuela, la universidad, el hogar, el lugar de trabajo, los espacios de ocio, etc.). Y esta ubicuidad aparentemente sin límites de las TIC”. (Riascos, Quintero, \& Ávila, 2009). 
Junto con otros factores como la importancia del aprendizaje a lo largo de la vida o la aparición de nuevas necesidades formativas, está en la base tanto de la aparición de nuevos escenarios educativos como de los profundos procesos de transformación.

Según (Coll \& Monereo, 2008) "Los niveles de uso de las TIC en el entorno escolar son extremadamente bajos, hasta el punto de que no pueden equipararse a los que los propios alumnos desarrollan fuera del entorno escolar, por lo menos en los países de la OCDE, y probablemente en un número creciente de Estados latinoamericanos. Las cifras disponibles arrojan un balance pobre y muy alejado de las expectativas iniciales. Es posible que tanto las ratios de alumnos por ordenador como las condiciones de los equipamientos, como la inevitable obsolescencia de una parte del parque instalado, sean razones de peso, pero probablemente no las únicas". Todo parece indicar, por lo tanto, que el desfase constatado entre expectativas y realidades no puede atribuirse únicamente a problemas de acceso.

Ciertamente, el acceso del profesorado y del alumnado a las TIC es una condición necesaria que está aún lejos de cumplirse en muchos países, muchas universidades y muchas aulas, por lo que es imprescindible seguir haciendo esfuerzos en este sentido.

\section{METODOLOGÍA}

La metodología que se utilizó en la investigación se inserta en un estudio bibliográfico, de tipo documental, de carácter descriptivo. De acuerdo con (Arias, 2006), "El nivel de investigación se refiere al grado de profundidad con que se aborda un objeto o fenómeno, y este puede ser exploratorio, descriptivo o explicativo"

Este estudio ofrece una aproximación al objeto de estudio, mediante una visión general de las tendencias de utilización de las herramientas telemáticas para la enseñanza en los Entornos Virtuales de Enseñanza Aprendizaje, y la descripción de elementos de análisis de estos, a partir de los datos aportados por los docentes. La aproximación resulta parcial, dado que desde la visión del estudiante podría verse complementada.

Se trata de una práctica descriptiva de las variables de estudio que tiene como propósito identificar las tendencias de uso de las diferentes estrategias y herramientas de enseñanza.

Los participantes fueron docentes de las diferentes carreras de una universidad pública de Ecuador que disponen de las bondades que ofrece el campus virtual Moodle

Este estudio analiza la problemática de la incorporación de las TIC al entorno de enseñanzaaprendizaje universitaria. Ello supone estudiar cómo las TIC se insertan en las prácticas educativas a fin de reflexionar acerca de cómo pueden mejorarlas, asumiendo que el aprendizaje depende, al menos en una parte importante, de la calidad de las prácticas 
desarrolladas en los Entornos Virtuales de Enseñanza Aprendizaje EVA y de las formas en que las herramientas son utilizadas por los docentes.

\section{RESULTADOS}

Se realizaron 293 encuestas online, las cuales arrojaron los resultados descritos a continuación.

Pregunta 1. Según su criterio la institución cuenta con infraestructura tecnología disponible:

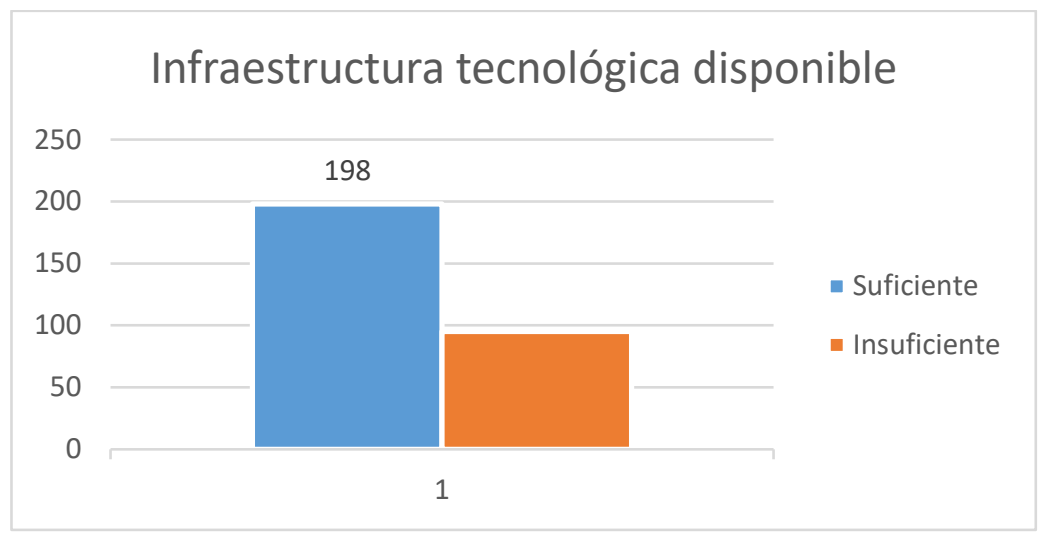

Gráfico 1. Infraestructura tecnológica disponible Fuente: Elaboración propia

Interpretación: Al analizar esta pregunta se puede evidenciar que el $68 \%$ de los docentes responde que la institución posee suficiente infraestructura tecnológica disponible, y el $32 \%$ de los docentes cree que la infraestructura tecnológica es insuficiente.

Pregunta 2. Qué recursos de las TICs son los que usted utiliza a menudo en clase.

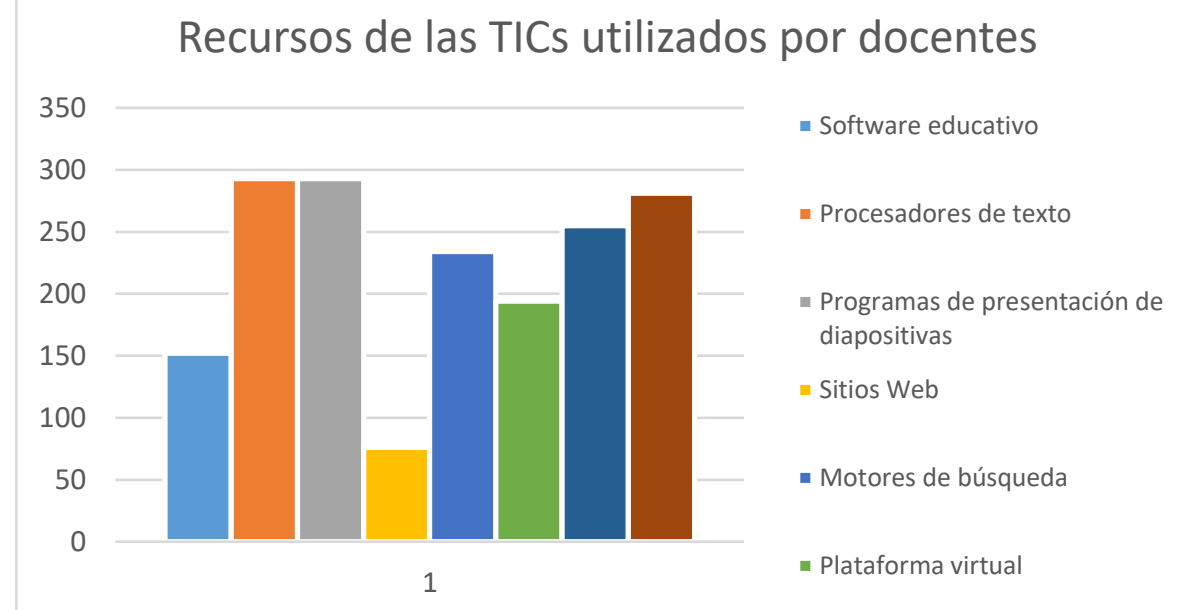

Gráfico 2. Recursos TICs utilizados por los docentes

Fuente: Elaboración propia

Interpretación: En el gráfico 2 se puede evidenciar que los docentes utilizan en un 100\% procesadores de texto y programas de presentación de diapositivas, seguidos de un $96 \%$ que 
utilizan redes sociales y un $87 \%$ utilizan el mail, y tan solo un $66 \%$ de los docentes utilizan la Plataforma virtual, por lo tanto, es necesario incentivar al docente sobre el uso de la plataforma virtual, ya que tiene muchas bondades.

Pregunta 3. ¿A cuántos cursos de capacitación técnica asiste usted en el año a través de la plataforma virtual?

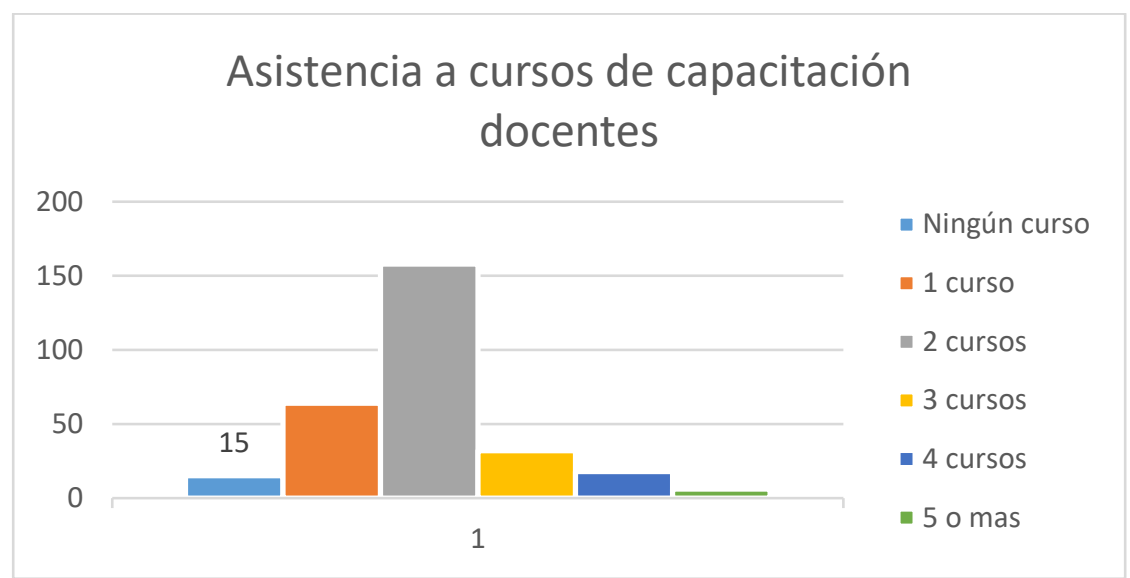

Gráfico 3. Asistencia a cursos virtuales de capacitación docentes Fuente: Elaboración propia

Interpretación: Analizando la frecuencia participativa de los docentes encuestados en cursos de capacitación, se advierte que el mayor porcentaje (54\%) participó en dos cursos de capacitación. Si bien revela un interés moderado de los docentes por la capacitación técnica, el desarrollo acelerado de las tecnologías educativas demanda una mayor dedicación a la observada.

Pregunta 4. Usted realiza foros o debates como un instrumento para desarrollar el aprendizaje

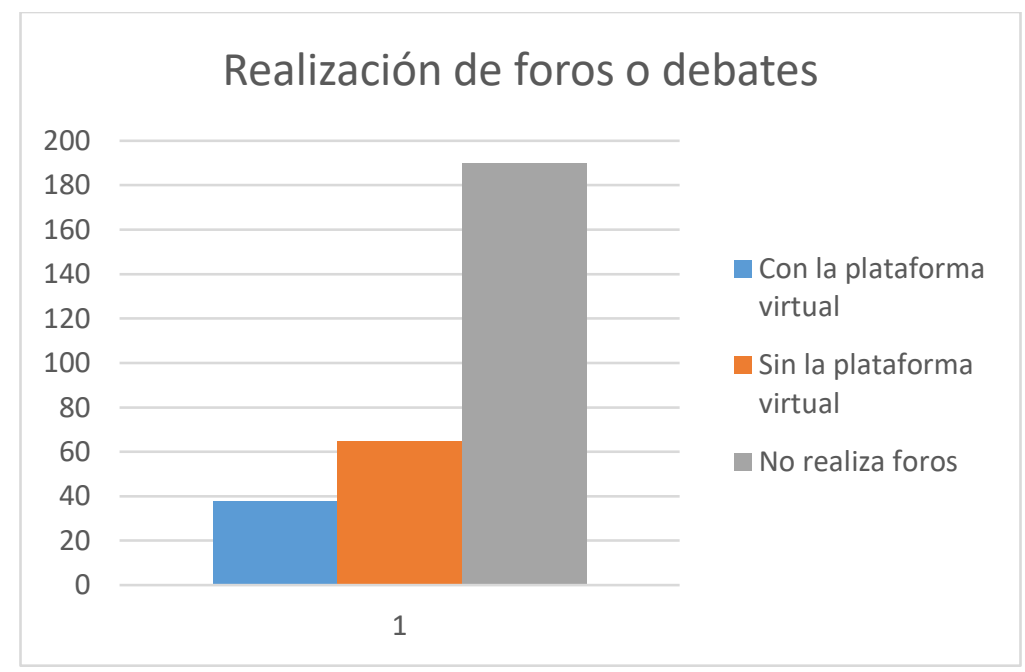

Gráfico 4. Realización de foros o debates Fuente: Elaboración propia 
Interpretación: Si bien el grado de utilización de los foros o debates es reducido, considerando que el $65 \%$ de los docentes no lo usan, sólo el $22 \%$ realizan esta actividad sin plataforma virtual y el $13 \%$ con plataforma virtual, lo que no guarda relación con la naturaleza de la actividad y evidencia una necesidad de revertir la tendencia al considerar que las bondades de la plataforma pueden agilizar este tipo de actividad.

Pregunta 5. Usted promueve los estudios de casos, la resolución de problemas y los trabajos por proyectos.

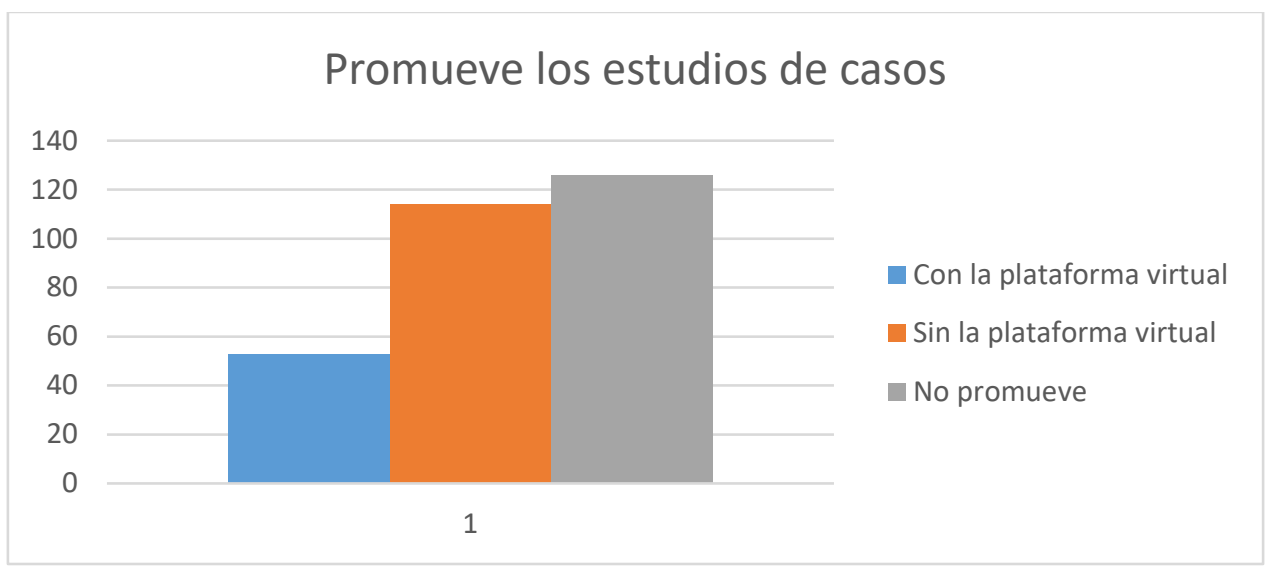

Gráfico 5. Promueve el estudio de casos, la resolución de problemas.

Fuente: Elaboración propia

Interpretación: Los estudios de casos, la resolución de problemas y los trabajos por proyectos reflejan poca utilización mientras que las menores frecuencias de empleo de estas estrategias tienden a ubicarse fuera de la plataforma virtual $42 \%$ y $20 \%$ con la plataforma virtual.

Pregunta 6. Usted promueve la elaboración de trabajos colaborativos:

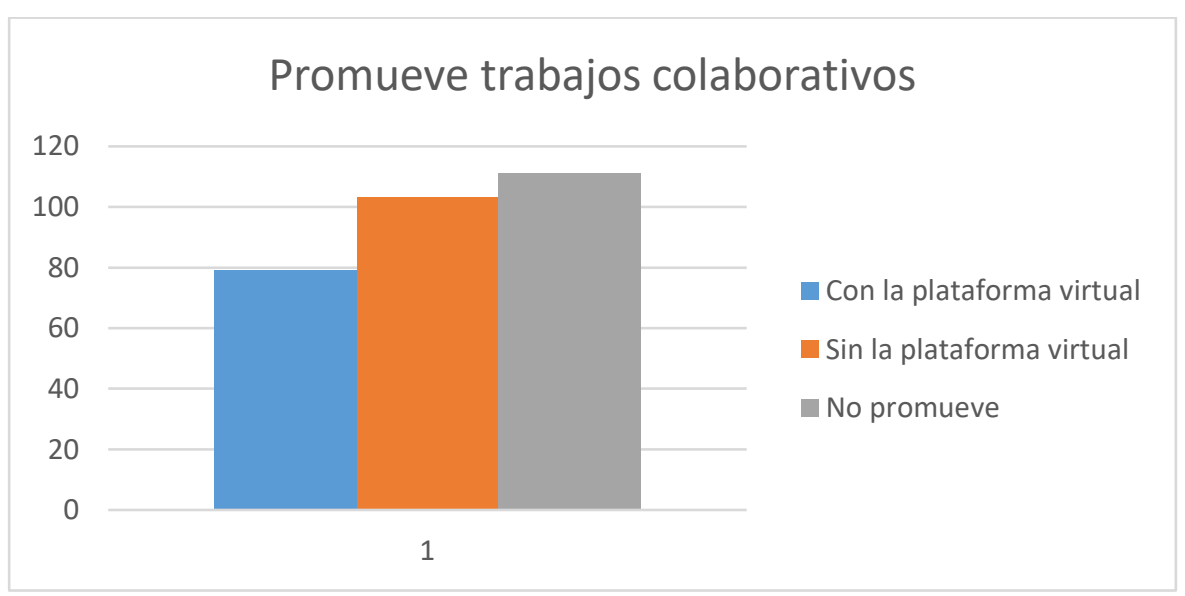

Gráfico 6. Promueve la elaboración de trabajos colaborativos

Fuente: Elaboración propia 
Interpretación: Por su parte los docentes en un 38\% no suelen promover la elaboración de trabajos colaborativos, el $35 \%$ realiza esta actividad sin el uso de la plataforma, y solo el $27 \%$ de los docentes promueve el trabajo colaborativo (Wiki) a través de la plataforma virtual. Cabe señalar que, aunque se da esta tendencia, las experiencias de aprendizaje colaborativo suelen cobrar mayor relevancia en entornos virtuales.

Pregunta 7. Usted realiza el proceso de autoevaluación luego del desarrollo de una clase.

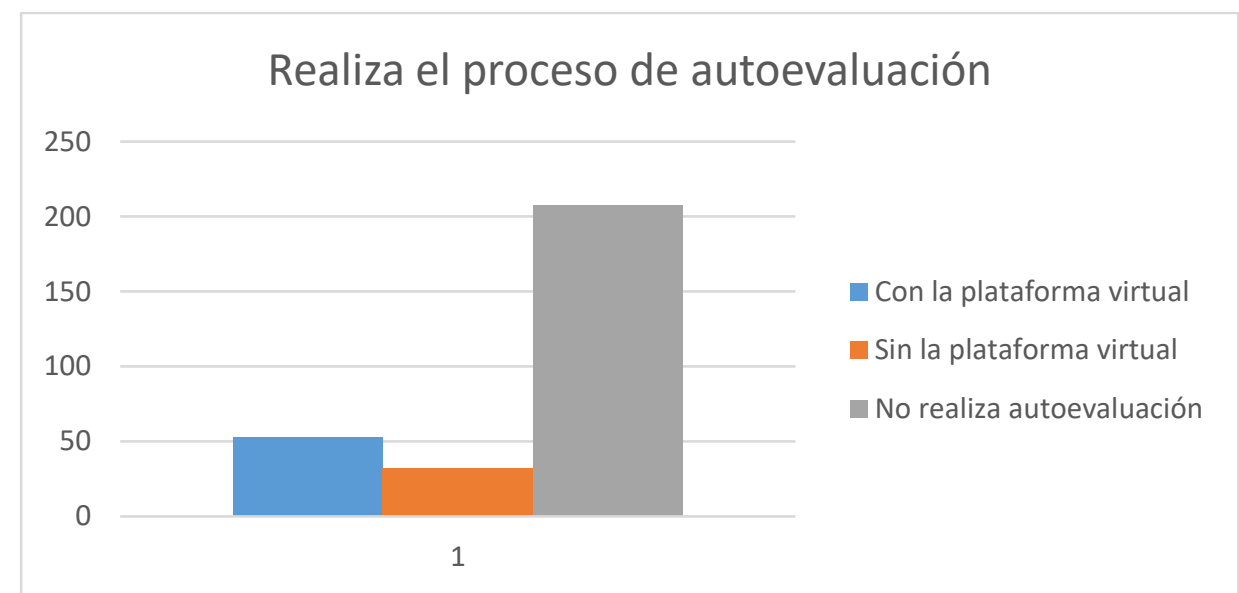

Gráfico 7. Realiza el proceso de autoevaluación al final de la clase Fuente: Elaboración propia

Interpretación: En relación con los ejercicios de autoevaluación son poco frecuentes, aunque curiosamente el mayor porcentaje de quienes lo utilizan siempre lo hacen en mayor proporción con la utilización de la plataforma virtual el 18\%, y sin la Plataforma lo realizan el $11 \%$, mientras que quienes no realizan esta actividad son el $71 \%$ de docentes.

\section{Conclusiones.}

- Los resultados permiten concluir que existe una predisposición de uso de técnicas y herramientas de aprendizaje, acorde a la intención de provocar la creatividad y la innovación en el ejercicio formativo, que se relaciona con las áreas disciplinares del desempeño docente. Se observa una mayor propensión a explorar las herramientas tecnológicas en aquellas disciplinas que invierten esfuerzos en la capacitación docente y en la incorporación de estas tecnologías.

- Se observa una escaza participación docente, en cursos de capacitación, lo que se refleja en la baja utilización de las herramientas tecnológicas que coadyuvaría a potenciar las estrategias de aprendizaje de los estudiantes. Así mismo la escaza utilización de estrategias sostenidas por la Plataforma Virtual, en contraste con la aplicación de las estrategias sin estas herramientas, amerita un análisis reflexivo del docente sobre las posibles causas del fenómeno observado. La implementación de las estrategias en cuestión, provistas desde la Plataforma Virtual, facilitaría el 
proceso de formación de los estudiantes, de ahí se puede especular que la capacitación es la base fundamental para que el docente explote acertadamente las bondades de las TICs en los entornos de enseñanza- aprendizaje.

- Finalmente se evidencia el trabajo de los docentes de provocar el análisis reflexivo y crítico de los estudiantes, actividad que podría verse favorecida con la mediación de las tecnologías de la comunicación y la información, ofrecidas por la plataforma virtual Moodle, tales como los portafolios, los WebQuest, los foros y los aportes colaborativos de las Wiki, entre otros, cuyas funcionalidades y utilidades no han sido aun suficientemente explotadas.

\section{Referencias Bibliográficas.}

Adell, J. (1997). Tendencias en educación en la sociedad de las tecnologías de la información. Revista Electrónica de Tecnología Educativa.

Adelle. (1997). Preparando para un nuevo modo de conocer. Desenvolupament de capacitats, 17.

Bosco, A. (1995). Las TIC en los procesos de convergencia europea y la innovación en la universidad: Las TIC en los procesos de convergencia europea y la innovación en la universidades: oportunidades y limitaciones. Aula Abierta, 24.

Cabero, J. (1996). Nuevas tecnologías, comunicación y educación. Revista Electronica de Tecnología y Educacion.

Coll, C., \& Monereo, C. (2008). Psicología de la Educación virtual: Aprender a enseñar con las tecnologías de la información y la comunicación. Madrid: Morata.

Collins, A. (1998). El potencial de las tecnologías de la información para la educación. Madrid: Ediciones Pirámide.

Comps, R. (1995). Libro Blanco sobre la Educación y la Formación. Santillana.

Heinze, G., Olmedo, V., \& Andoney, J. (2017). Uso de las tecnologías de la información y comunicación en las residencias en México. Ensayos y opiniones. Obtenido de http://www.medigraphic.com/pdfs/actmed/am-2017/am172p.pdf

ISTE. (1992). Guidelines for Accreditation of Educatinal Computing and Technology Programs. Accreditation and Standards Committee.

Kay, R., \& Knaack, L. (2009). Assessing learning, quality and engagement in learning objects: the Learning Object Evaluation Scale for Students (LOES-S). Education Tech Research, 21. Obtenido de http://web.ebscohost.com/ehost/pdfviewer/pdfviewer?

Marina, J. (1995). El timo de la sociedad de la información. Madrid: Santillana. 
Mata, F. (1997). Dificultades en el aprendizaje de la expresión escrita. Aljibe.

Organización de las Naciones Unidas para la Educación la Ciencia y la Cultura. (2017). Las

Tecnologías de la Información y la Comunicación (TIC) en la Educación. Revista metropolitana de ciencias aplicadas. Obtenido de http://www.unesco.org/new/es/havana/areas-of-action/education/tic-en-la-educacion/

Palomo, R., Ruiz, J., \& Sánchez, J. (2010). Enseñanza con TIC en el siglo XXI. La escuela 2.0. Educatio Siglo XXI. Obtenido de https://revistas.um.es/educatio/article/view/112071/106391

Poole, B. (1999). Tecnología educativa. Madrid: Mc Graw Hill.

Prieto, V., Quiñones, I., Ramírez, G., Fuentes, Z., Labrada, T., \& Pérez, O. (2011). Impacto de las tecnologías de la información y las comunicaciones en la educación y nuevos paradigmas del enfoque educativo. Educación media superior, 95-102.

Puig, R., \& Ramos, P. (2009). Consideraciones generales de los métodos de enseñanza y su aplicación en cada etapa del aprendizaje. Revista Habana ciencias médicas. Obtenido de http://scielo.sld.cu/scielo.php?script=sci_arttext\&pid=S1729-519X2009

Riascos, S., Quintero, D., \& Ávila, G. (2009). Las TIC en el aula: percepciones de los profesores universitarios. Educacion y Educadores.

Ruiz, E. (2012). El aprendizaje colaborativo escenario para la construcción social de aprendizajes significativos. Cognición, 8(38). Obtenido de http://www.cognicion.net/images/articulos/Cog38/38-2-el-aprendizaje-colaborativoescenario-para-la-construccion-social-de-aprendizajes-significativos

Salinas, J. (2008). Modelos didácticos en los campus virtuales universitarios: patrones metodológicos generados por los profesores en procesos de enseñanza aprendizaje en entornos virtuales". ResearchGate. Obtenido de https://www.researchgate.net/publication/263729982_Modelos_didacticos_en_los_camp us_virtuales_universitarios_Perfiles_metodologicos_de_los_profesores_en_procesos_de_ ensenanza-aprendizaje_en_entornos_virtuales

Sculley, J. (1999). Qué se entiende por una institución de educación superior flexible. 167.

Suasnabas, L., Díaz, E., Avila, W., \& Rodríguez, V. (2017). Las Tics en los procesos de enseñanza y aprendizaje en la educación universitaria. Dominio de las ciencias, 721-749. 


\section{PARA CITAR EL ARTÍCULO INDEXADO.}

Gavilanes Sagñay, M., Yanza Chavez, W. G., Inca Falconi, A., Torrez Guananga, G., \& Sánchez Chávez, R. (2019). Las TICs en los procesos de enseñanza y aprendizaje. Ciencia Digital, 3(2.6), 422-439. https://doi.org/10.33262/cienciadigital.v3i2.6.575

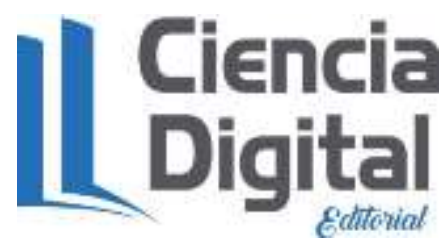

El artículo que se publica es de exclusiva responsabilidad de los autores y no necesariamente reflejan el pensamiento de la Revista Ciencia Digital.

El artículo queda en propiedad de la revista y, por tanto, su publicación parcial y/o total en otro medio tiene que ser autorizado por el director de la Revista Ciencia Digital.
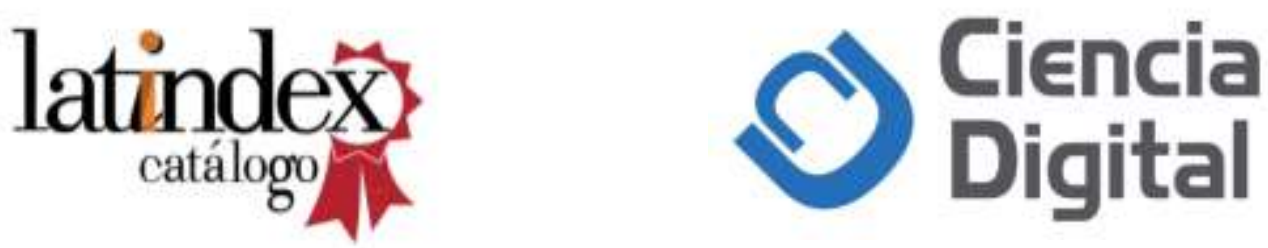